\title{
FUZZY SOFT IDEAL, FUZZY SOFT BI-IDEAL, FUZZY SOFT QUASI-IDEAL AND FUZZY SOFT INTERIOR IDEAL OVER ORDERED $\Gamma$-SEMIRING
}

\author{
MARAPUREDDY MURALI KRISHNA RAO*
}

Department of Mathematics, GIT, GITAM University, Visakhapatnam, Andhra Pradesh, India-530 045

*Corresponding author: mmarapureddy@gmail.com

Received Nov 18, 2017

\begin{abstract}
In this paper, we introduce the notion of fuzzy soft ordered $\Gamma$-semiring, fuzzy soft ideal,fuzzy soft bi-ideal,fuzzy soft quasi-ideal and fuzzy soft interior ideal over ordered $\Gamma$-Semirings and study some of their algebraical properties and relations between them. 2010 Mathematics Subject Classification.16Y60, 08A72.

Key words and phrases. ordered $\Gamma$-Semiring, fuzzy soft $\Gamma$-semiring, fuzzy soft ideal, fuzzy soft bi-ideal,fuzzy soft quasi-ideal, fuzzy soft interior ideal.
\end{abstract}

\section{INTRODUCTION}

The notion of $\Gamma$-semiring was introduced by Murali Krishna Rao [19,20,21,26] in 1995, not only generalizes the notion of semiring and $\Gamma$-ring but also the notion of ternary semiring. The notion of $\Gamma$-ring was introduced by Nobusawa [32] as a generalization of ring in 1964. Sen [34] introduced the notion of $\Gamma$-semigroup in 1981. The notion of ternary algebraic system was introduced by Lehmer [14] in 1932, Lister [15] introduced ternary ring. Dutta \& Kar [3] introduced the notion of ternary semiring which is a generalization of ternary ring and semiring. Semiring is an algebraic structure which is a common generalization of rings and distributive lattices, was first introduced by Vandiver [37] in 1934 but non-trivial examples of semirings had appeared in the studies on the theory of commutative ideals of rings by Dedekind in 19th century. Semiring is a universal algebra with two binary operations called addition and multiplication, where one of them distributive over the other. Bounded distributive lattices are commutative semirings which are both additively idempotent and multiplicatively idempotent. A natural example of semiring is the set of all natural numbers under usual addition and multiplication of numbers. In particular, if $I$ is the unit interval on the real line, then $(I, \max , \min )$ is a semiring in which 0 is the additive identity and 1 is the mutiplicative identity. The theory of rings and the theory of semigroups have considerable 
impact on the development of the theory of semirings. In structure, semirings lie between semigroups and rings. Additive and multiplicative structures of a semiring play an important role in determining the structure of a semiring. Semiring as the basic algebraic structure was used in the areas of theoretical computer science as well as in the solutions of graph theory, optimization theory and in particular for studying automata, coding theory and formal languages. Semiring theory has many applications in other branches. The notion of ideals was introduced by Dedekind for the theory of algebraic numbers, was generalized by E. Noether for associative rings. The one and two sided ideals introduced by her, are still central concepts in ring theory. We know that the notion of an one sided ideal of any algebraic structure is a generalization of notion of an ideal. The quasi ideals are generalization of left and right ideals where as the bi-ideals are generalization of quasi ideals. Herniksen [7] defined $k$-ideals in semirings to obtain analogous of ring results. The concept of bi-ideals was first introduced by R.A. Good and D.R. Hughes[6] for a semigroup. As a further generalization of ideals, Steinfeld [35] first introduced the notion of quasi ideals for semigroups and then for rings. Iseki $[8,9,10]$ introduced the concept of quasi ideal for a semiring and studied ideals in semirings. We know that the notion of the bi-ideal in semirings is a special case of $(\mathrm{m}, \mathrm{n})$ ideal introduced by S. Lajos[12]. Lajos and Szasz [13] introduced the concept of bi-ideals for rings.Quasi ideals in $\Gamma$-semirings studied by Jagtap and Pawar[11].In 2017,Marapureddy Murali Krishna Rao [25,26,28,30] introduced, bi-quasi-ideals in semirings bi-quasi-ideals and fuzzy bi-quasi-ideals in $\Gamma$-semigroups and $T$ - fuzzy ideals in ordered $\Gamma$-semirings.

The theory of fuzzy sets is the most appropriate theory for dealing with uncertainty is first introduced by Zadeh [38,39]. The fuzzification of algebraic structure was introduced by A. Rosenfeld [33] and he introduced the notion of fuzzy subgroups in 1971.Dutta et al.[2]studied fuzzy ideals in $\Gamma$-semirings..Mandal [17]studied fuzzy ideals and fuzzy interior ideals in ordered semirings Swamy and Swamy [36]studied fuzzzy prime ideals of rings. Molodtsov [18] introduced the concept of soft set theory as a new mathematical tool for dealing with uncertainties, only partially resolves the problem is that objects in universal set often does not precisely satisfy the parameters associated to each of the elements in the set. Then Maji et al.[16] extended soft set theory to fuzzy soft set theory. Aktas and Cagman defined the soft set and soft groups. Majumdar and Samantha extended soft sets to fuzzy soft set. Acar et al. [1] gave the basic concept of soft ring. Jayanth Ghosh et al. [5] initiated the study of fuzzy soft rings and fuzzy soft ideals. Feng et al. [4] studied soft semirings by using the soft set theory M.Murali Krishna Rao [22,23,32] introduced and studied fuzzy soft ideals and fuzzy soft $k$-ideals over a $\Gamma$-semiring and a semiring In this paper, we introduce the notion of fuzzy soft ordered $\Gamma$-semiring, fuzzy soft ideal,fuzzy soft bi-ideal,fuzzy soft 
quasi-ideal and fuzzy soft interior ideal over ordered $\Gamma$-semirings and study some of their algebraical properties.

\section{Preliminaries}

In this section, we recall some definitions introduced by the pioneers in this field earlier.

Definition 2.1. A set $M$ together with two associative binary operations called addition and multiplication (denoted by + and $\cdot$ respectively) will be called

semiring provided

(i) addition is a commutative operation.

(ii) there exists $0 \in M$ such that $x+0=x$ and $x \cdot 0=0 \cdot x=0$ for each $x \in M$.

(iii) multiplication distributes over addition both from the left and from the right.

Definition 2.2. Let $(M,+)$ and $(\Gamma,+)$ be commutative semigroups. Then we call $M$ as a $\Gamma$-semiring, if there exists a mapping $M \times \Gamma \times M \rightarrow M$ written as $(x, \alpha, y)$ as xay such that it satisfies the following axioms for all $x, y, z \in M$ and $\alpha, \beta \in \Gamma$

(i) $x \alpha(y+z)=x \alpha y+x \alpha z$

(ii) $(x+y) \alpha z=x \alpha z+y \alpha z$

(iii) $x(\alpha+\beta) y=x \alpha y+x \beta y$

(iv) $x \alpha(y \beta z)=(x \alpha y) \beta z$.

Definition 2.3. A $\Gamma$-semiring $M$ is called an ordered $\Gamma$-semiring if it admits a compatible relation $\leq$. i.e. $\leq$ is a partial ordering on $M$ satisfying the following conditions. If $a \leq b$ and $c \leq d$ then

(i) $a+c \leq b+d$ (ii) $a \alpha c \leq b \alpha d$ (iii) $c \alpha a \leq d \alpha b$, for all $a, b, c, d \in M, \alpha \in \Gamma$.

Example 2.4. Let $M=[0,1], \Gamma=N$, , binary operation be defined as $x+y=\max \{x, y\}$, and ternary operation be defined as $x \gamma y=\min \{x, \gamma, y\}$ for all $x, y \in M, \gamma \in \Gamma$. Then $M$ is an ordered $\Gamma$-semiring with respect to usual ordering.

Definition 2.5. Let $M$ be an ordered $\Gamma$-semiring and $A$ be a non-empty subset of $M$. $A$ is called a $\Gamma$-subsemiring of ordered $\Gamma$-semiring $M$ if $A$ is a sub-semigroup of $(M,+)$ and $A \Gamma A \subseteq A$.

Definition 2.6. Let $M$ be an ordered $\Gamma$-semiring. A non-empty subset $A$ of $M$ is called a left (right) ideal of an ordered $\Gamma$-semiring $M$ if $A$ is closed under addition and $M \Gamma A \subseteq$ $A(A \Gamma M \subseteq A)$ and for any $a \in M, b \in A, a \leq b$ then $a \in A$. $A$ is called an ideal of $M$ if it is both a left ideal and a right ideal.

Definition 2.7. A fuzzy subset $\mu: M \rightarrow[0,1]$ is non-empty if $\mu$ is not the constant function. 
Definition 2.8. For any two fuzzy subsets $\lambda$ and $\mu$ of $M, \lambda \subseteq \mu$ means $\lambda(x) \leq \mu(x)$ for all $x \in M$.

Definition 2.9. Let $U$ be an initial universe set and $E$ be the set of parameters. Let $P(U)$ denotes the power set of $U$. A pair $(f, E)$ is called soft set over $U$ where $f$ is a mapping given by $f: E \rightarrow P(U)$.

Definition 2.10. For a soft set $(f, A)$, the set $\{x \in A \mid f(x) \neq \emptyset\}$ is called Support of $(f, A)$, denoted by $\operatorname{Supp}(f, A)$. If $\operatorname{Supp}(f, A) \neq \emptyset$ then $(f, A)$ is called a non null soft set.

Definition 2.11. Let $U$ be an initial universe set, $E$ be the set of parameters and $A \subseteq E$. $A$ pair $(f, A)$ is called fuzzy soft set over $U$ where $f$ is a mapping given by $f: A \rightarrow I^{U}$ where $I^{U}$ denotes the collection of all fuzzy subsets of $U$.

Definition 2.12. Let $(f, A),(g, B)$ be fuzzy soft sets over $U$. Then $(f, A)$ is said to be fuzzy soft subset of $(g, B)$ denoted by $(f, A) \subseteq(g, B)$ if $A \subseteq B$ and $f(a) \subseteq g(a)$ for all $a \in A$.

\section{Main Results}

In this section,we introduce the concepts of soft ordered $\Gamma$-semiring,fuzzy soft ordered $\Gamma$-Semiring, fuzzy soft bi-ideal,fuzzy soft interior ideal and fuzzy soft quasi-ideal over ordered $\Gamma$-semiring, study the properties related to these notions and relations between them.

Definition 3.1. Let $M$ be an ordered $\Gamma$-semiring, $E$ be a parameter set and $A \subseteq E$. Let $f$ be a mapping given by $f: A \rightarrow P(M)$ where $P(M)$ is the power set of $M$. Then $(f, A)$ is called a soft ordered $\Gamma$-semiring over $M$ if and only if for each $a \in A, f(a)$ is $\Gamma$-subsemiring of $M$. i.e.

(i) $x, y \in M \Rightarrow x+y \in f(a)$

(ii) $x, y \in M, \alpha \in \Gamma \Rightarrow x \alpha y \in f(a)$ for all $x, y \in M, \alpha \in \Gamma$.

Definition 3.2. Let $M$ be an ordered $\Gamma$-semiring, $E$ be a parameter set and $A \subseteq E$. Let $f$ be a mapping given by $f: A \rightarrow[0,1]^{M}$ where $[0,1]^{S}$ denotes the collection of all fuzzy subsets of $M$. Then $(f, A)$ is called a fuzzy soft $\Gamma$-semiring over $M$ if and only if for each $a \in A, f(a)=f_{a}$ is the fuzzy $\Gamma$-subsemiring of $M$. i.e.,

(i) $f_{a}(x+y) \geq \min \left\{f_{a}(x), f_{a}(y)\right\}$

(ii) $f_{a}(x \alpha y) \geq \min \left\{f_{a}(x), f_{a}(y)\right\}$ for all $x, y \in M, \alpha \in \Gamma$.

Definition 3.3. Let $M$ be an ordered $\Gamma$-semiring, $E$ be a parameter set and $A \subseteq E$. Let $f$ be a mapping given by $f: A \rightarrow P(M)$. Then $(f, A)$ is called a soft left(right) ideal over $M$ if and only if for each $a \in A, f(a)$ is a left(right) ideal of M. i.e.,

(i) $x, y \in f(a) \Rightarrow x+y \in f(a)$ 
(ii) $x, y \in f(a), \alpha \in \Gamma, r \in M \Rightarrow \operatorname{r\alpha x}(x \alpha r) \in f(a)$.

(iii) $x \leq y \Rightarrow f_{a}(x) \geq f_{a}(y)$,for all $x, y \in M, \alpha, \beta \in \Gamma$.

$(f, A)$ is called a soft ideal over of $M$ if for each $a \in A, f(a)$ is both a left ideal and a right ideal.

Definition 3.4. Let $M$ be an ordered $\Gamma$-semiring, $E$ be a parameter set and $A \subseteq E$. Let $f$ be a mapping given by $f: A \rightarrow[0,1]^{M}$ where $[0,1]^{M}$ denotes the collection of all fuzzy subsets of $M$. Then $(f, A)$ is called a fuzzy soft left(right) ideal over $M$ if and only if for each $a \in A$, the corresponding fuzzy subset $f_{a}: S \Rightarrow[0,1]$ is a fuzzy left(right) ideal of $M$. i.e.,

(i) $f_{a}(x+y) \geq \min \left\{f_{a}(x), f_{a}(y)\right\}$

(ii) $f_{a}(x \alpha y) \geq f_{a}(y)\left(f_{a}(x)\right)$

(iii) $x \leq y \Rightarrow f_{a}(x) \geq f_{a}(y)$,for all $x, y \in M, \alpha, \beta \in \Gamma$.

Definition 3.5. Let $M$ be an ordered $\Gamma$-semiring, $E$ be a parameter set and $A \subseteq E$. Let $f$ be a mapping given by $f: A \rightarrow[0,1]^{M}$ where $[0,1]^{M}$ denotes the collection of all fuzzy subsets of $M$. Then $(f, A)$ is called a fuzzy soft ideal over $M$ if and only if for each a $\in A$, the corresponding fuzzy subset $f_{a}: M \rightarrow[0,1]$ is a fuzzy ideal of $M$. i.e.,

(i) $f_{a}(x+y) \geq \min \left\{f_{a}(x), f_{a}(y)\right\}$

(ii) $f_{a}(x \alpha y) \geq \max \left\{f_{a}(x), f_{a}(y)\right\}$

(iii) $x \leq y \Rightarrow f_{a}(x) \geq f_{a}(y)$, for all $x, y \in M, \alpha, \beta \in \Gamma$.

Definition 3.6. Let $M$ be an ordered $\Gamma$-semiring, $E$ be a parameter set and $A \subseteq E$. Let $f$ be a mapping given by $f: A \rightarrow[0,1]^{M}$ where $[0,1]^{M}$ denotes the collection of all fuzzy subsets of $M$. Then $(f, A)$ is called a fuzzy soft bi-ideal over $M$ if and only if for each $a \in A$, the corresponding fuzzy subset $f_{a}: M \rightarrow[0,1]$ is a fuzzy bi-ideal of $M$. i.e.,

(i) $f_{a}(x+y) \geq \min \left\{f_{a}(x), f_{a}(y)\right\}$

(ii) $f_{a}(x \alpha y \beta z) \geq \max \left\{f_{a}(x), f_{a}(z)\right\}$ for all $x, y \in M, \alpha, \beta \in \Gamma$.

(iii) $x \leq y \Rightarrow f_{a}(x) \geq f_{a}(y)$,for all $x, y, z \in M, \alpha, \beta \in \Gamma$.

Definition 3.7. Let $M$ be an ordered $\Gamma$-semiring, $E$ be a parameter set and $A \subseteq E$. Let $f$ be a mapping given by $f: A \rightarrow[0,1]^{M}$ where $[0,1]^{M}$ denotes the collection of all fuzzy subsets of $M$. Then $(f, A)$ is called a fuzzy soft interor ideal over $M$ if and only if for each $a \in A$, the corresponding fuzzy subset $f_{a}: M \rightarrow[0,1]$ is a fuzzy interior ideal of $M$. i.e.,

(i) $f_{a}(x+y) \geq \min \left\{f_{a}(x), f_{a}(y)\right\}$

(ii) $f_{a}(x \alpha y \beta z) \geq \max \left\{f_{a}(y)\right\}$

(iii) $x \leq y \Rightarrow f_{a}(x) \geq f_{a}(y)$, for all $x, y, z \in M, \alpha, \beta \in \Gamma$. 
Definition 3.8. Let $M$ be an ordered $\Gamma$-semiring, $E$ be a parameter set and $A \subseteq E$. Let $f$ be a mapping given by $f: A \rightarrow[0,1]^{M}$ where $[0,1]^{M}$ denotes the collection of all fuzzy subsets of $M$. Then $(f, A)$ is called a fuzzy soft quasi ideal over $M$ if and only if for each $a \in A$, the corresponding fuzzy subset $f_{a}: M \rightarrow[0,1]$ is a fuzzy quasi ideal of $M$. i.e.A fuzzy subset $f_{a}$ of ordered $\Gamma$-semiring $M$ is called a fuzzy quasi ideal i.e.

(i) $f_{a}(x+y) \geq \min \left(f_{a}(x), f_{a}(y)\right)$ (ii) $f_{a} \circ \chi_{M} \wedge \chi_{M} \circ f_{a} \subseteq f_{a}$

(iii) $x \leq y \Rightarrow f_{a}(x) \geq f_{a}(y)$, for all $x, y \in M$.

Definition 3.9. Let $(f, A)$ and $(g, B)$ be fuzzy soft sets over an ordered $\Gamma$-semiring $M$. The intersection of fuzzy soft sets $(f, A)$ and $(g, B)$ is denoted by $(f, A) \cap(g, B)=(h, C)$ where $C=A \cup B$ is defined as

$$
h_{c}= \begin{cases}f_{c}, & \text { if } c \in A \backslash B ; \\ g_{c}, & \text { if } c \in B \backslash A ; \\ f_{c} \cap g_{c}, & \text { if } c \in A \cap B .\end{cases}
$$

for all $c \in A \cup B$ and $x \in M$.

Definition 3.10. Let $(f, A)$ and $(g, B)$ be fuzzy soft sets over an ordered $\Gamma$-semiring $M$. The union of fuzzy soft sets $(f, A)$ and $(g, B)$ is denoted by $(f, A) \cup(g, B)=(h, C)$ where $C=A \cup B$ is defined as

$$
h_{c}= \begin{cases}f_{c}, & \text { if } c \in A \backslash B ; \\ g_{c}, & \text { if } c \in B \backslash A ; \\ f_{c} \cup g_{c}, & \text { if } c \in A \cap B .\end{cases}
$$

for all $c \in A \cup B$ and $x \in M$.

Definition 3.11. Let $(f, A)$ and $(g, B)$ be fuzzy soft sets over an ordered $\Gamma$-semiring $S$. " $(f, A)$ and $(g, B)$ is denoted by " $(f, A) \wedge(g, B)$ " is defined by $(f, A) \wedge(g, B)=(h, C)$ where $C=A \times B . h_{c}(x)=\min \left\{f_{a}(x), g_{b}(x)\right\}$ for all $c=(a, b) \in A \times B$ and $x \in M$.

Definition 3.12. Let $(f, A)$ and $(g, B)$ be fuzzy soft sets over an ordered $\Gamma$-semiring $M$. " $(f, A)$ or $(g, B)$ " is denoted by $(f, A) \vee(g, B)$ is defined by $(f, A) \vee(g, B)=(h, C)$ where $C=A \times B$ and $h_{c}(x)=\max \left\{f_{a}(x), g_{b}(x)\right\}$ for all $c=(a, b) \in A \times B, x \in U$.

Definition 3.13. let $M$ be an ordered $\Gamma$ - semiring and $x \in M$.

We define $M_{x}=\{(y, z) \in M \times M / x \leq y \alpha z, \alpha \in \Gamma\}$. For any fuzzy subset $\mu$ and $\gamma$ of $M$, $\mu \circ \gamma: M \rightarrow[0,1]$ is defined by

$$
\mu \circ \gamma(x)= \begin{cases}\sup _{(y, z) \in M_{x}} & \{\min (\mu(y), \gamma(z))\}, \text { if } M_{x} \neq \phi . \\ 0, & \text { otherwise. }\end{cases}
$$


Definition 3.14. Let $(f, A)$ and $(g, B)$ be fuzzy soft ideals over an ordered $\Gamma$-semiring $M$. The product $(f, A)$ and $(g, B)$ is defined as $((f \circ g), C)$ where $C=A \cup B$ and

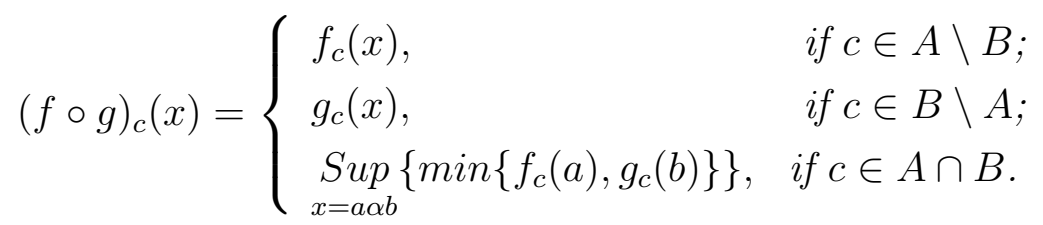

for all $c \in A \cup B$ and $x \in M, \alpha \in \Gamma$.

Theorem 3.15. Let $(f, A)$ and $(g, B)$ be fuzzy soft ordered $\Gamma$-semirings over an ordered $\Gamma$-semiring $M$. Then $(f, A) \cup(g, B)$ is a fuzzy soft ordered $\Gamma$-semiring over $M$.

Proof. Let $(f, A) \cup(g, B)=(h, C)$ where $C=A \cup B$.

Case $(i)$ : if $A \cap B=\emptyset$.

Let $c \in C=A \cup B$. Then $c \in A$ or $c \in B$. If $c \in A$ then $h_{c}=f_{c}$ and if $c \in B$ then $h_{c}=g_{c}$, since $(f, A)$ and $(g, B)$ are fuzzy soft $\Gamma$-semirings over a $\Gamma$-semiring $M$. In both cases $h_{c}$ is a fuzzy $\Gamma$-subsemiring of $M$.

Case (ii) : if $A \cap B \neq \emptyset$.

If $c \in A \backslash B$ then $h_{c}=f_{c}, h_{c}$ is a fuzzy $\Gamma$-subsemiring.

If $c \in B \backslash A$ then $h_{c}=g c, h_{c}$ is a fuzzy $\Gamma$-subsemiring.

If $c \in A \cap B$ then $h_{c}=f_{c} \cup g_{c}$.

Let $x, y \in M, \alpha \in \Gamma$. Then

$$
\begin{aligned}
h_{c}(x+y) & =f_{c} \cup g_{c}(x+y) \\
& =\max \left\{f_{c}(x+y), g_{c}(x+y)\right\} \\
& \geq \max \left\{\min \left\{f_{c}(x), f_{c}(y)\right\}, \min \left\{g_{c}(x), g_{c}(y)\right\}\right\} \\
& =\min \left\{\max \left\{f_{c}(x), g_{c}(x)\right\}, \max \left\{f_{c}(y), g_{c}(y)\right\}\right\} \\
& =\min \left\{f_{c} \cup g_{c}(x), f_{c} \cup g_{c}(y)\right\} \\
h_{c}(x \alpha y) & =f_{c} \cup g_{c}(x \alpha y) \\
& =\max \left\{f_{c}(x \alpha y), g_{c}(x \alpha y)\right\} \\
& \geq \max \left\{\min \left\{f_{c}(x), f_{c}(y)\right\}, \min \left\{g_{c}(x), g_{c}(y)\right\}\right\} \\
& =\min \left\{\max \left\{f_{c}(x), g_{c}(x)\right\}, \max \left\{f_{c}(y), g_{c}(y)\right\}\right\} \\
& =\min \left\{f_{c} \cup g_{c}(x), f_{c} \cup g_{c}(y)\right\}
\end{aligned}
$$

Hence $h_{c}$ is a fuzzy ordered $\Gamma$-subsemiring of $M$. Therefore $(f, A) \cup(g, B)$ is a fuzzy soft ordered $\Gamma$-semiring over $M$.

The proofs of the following theorems are similar to Theorem [3.15]. 
Theorem 3.16. Let $(f, A)$ and $(g, B)$ be fuzzy soft ordered $\Gamma$-semirings over an ordered $\Gamma$-semiring $M$. Then $(f, A) \cap(g, B)$ is a fuzzy soft ordered $\Gamma$-semiring over $M$.

Theorem 3.17. Let $(f, A)$ and $(g, B)$ be fuzzy soft ordered $\Gamma$-semirings over an ordered $\Gamma$-semiring $M$. Then $(f, A) \wedge(g, B)$ is a fuzzy soft ordered $\Gamma$-semiring over $M$.

Theorem 3.18. Let $(f, A)$ and $(g, B)$ be fuzzy soft ordered $\Gamma$-semirings over an ordered $\Gamma$-semiring $S$. Then $(f, A) \vee(g, B)$ is a fuzzy soft ordered $\Gamma$-semiring over $S$.

Definition 3.19. Let $(f, A)$ and $(g, B)$ be fuzzy soft ordered $\Gamma$-semirings over $M$. Then $(g, B)$ is a fuzzy soft ordered $\Gamma$-subsemiring of $(f, A)$ if it satisfies the following conditions (i) $B \subset A($ ii $) g_{b}(x) \leq f_{a}(x)$ for all $x \in \operatorname{Supp}(g, B)$.

Theorem 3.20. Let $M$ be an ordered $\Gamma$-semiring and $E$ be a parameterset and $A \subseteq E$. If $(f, A)$ is a fuzzy soft left(right) ideal over $M$ then if for each $a \in A$ then $\chi_{M} \circ f_{a} \subseteq$ $f_{a}\left(f_{a} \circ \chi_{M} \subseteq f_{a}\right)$, where $\chi_{M}$ stands for characteristic function of $M$.

Proof. Suppose $(f, A)$ is a fuzzy soft left ideal over $M$. Then, for each $a \in A, f_{a}$ is fuzzy left ideal of $M$. Let $z \in M$. Then

$$
\chi_{M} \circ f_{a}(z)=\sup _{z \leq x \alpha y}\left\{\min \left\{\chi_{M}(x), f_{a}(y)\right\}\right\}=\sup _{z \leq x \alpha y}\left\{f_{a}(y)\right\} \leq f_{a}(x \alpha y)=f_{a}(z)
$$

If $z$ cannot be expressed as $z \leq x \alpha y$ where $x, y \in M, \alpha \in \Gamma$ then $\chi_{S} \circ f_{a}(z)=0 \leq f_{a}(z)$. Similarly we can prove the result for a fuzzy soft right ideal over $M$.

Theorem 3.21. Let $M$ be an ordered $\Gamma$-semiring and $E$ be a parameterset and $A \subseteq E$. If $(f, A)$ is a fuzzy soft right ideal over $M$ then $(f, A)$ is a fuzzy soft quasi ideal over $M$

Proof. Suppose $(f, A)$ is a fuzzy soft right ideal over $M$. Then, for each $a \in A, f_{a}$ is a fuzzy right ideal of $M$. Let $x \in M$. and $x \leq u \alpha v, u, v \in S, \alpha \in \Gamma$. Then

$$
\begin{aligned}
f_{a}(x) & \geq f_{a}(u \alpha v) \\
& \geq f_{a}(u) \\
& =\min \left\{f_{a}(u), \chi_{M}(v)\right\} \\
& \geq \min \left(f_{a}(u), \chi_{M}(v)\right) . \\
\Rightarrow f_{a}(x) & \geq \sup _{(y, z) \in M_{x}}\left\{\min \left(f_{a}(u), \chi_{M}(v)\right)\right\} \\
& =f_{a} \circ \chi_{M}(x) .
\end{aligned}
$$

Therefore $f_{a}(x) \geq \min \left\{f_{a} \circ \chi_{M}(x), \chi_{M} \circ f_{a}(x)\right\}$

$$
\begin{aligned}
& \geq \min \left(f_{a} \circ \chi_{M}(x), \chi_{M} \circ f_{a}(x)\right) \\
& =\chi_{M} \circ f_{a} \wedge f_{a} \circ \chi_{M}(x) .
\end{aligned}
$$

Therefore $f_{a}$ is a fuzzy quasi ideal of $M$. Hence $(f, A)$ is a fuzzy soft quasi ideal over $M$ 
Corollary 3.22. Let $M$ be an ordered $\Gamma$-semiring and $E$ be a parameter set and $A \subseteq E$. If $(f, A)$ is a fuzzy soft left ideal over $M$ then $(f, A)$ is a fuzzy soft quasi ideal over $M$

Corollary 3.23. Let $M$ be an ordered $\Gamma$-semiring and $E$ be a parameter set and $A \subseteq E$. If $(f, A)$ is a fuzzy soft ideal over $M$ then $(f, A)$ is a fuzzy soft quasi ideal over $M$

Theorem 3.24. Let $(f, A)$ and $(g, B)$ be fuzzy soft bi-ideals over ordered $\Gamma$-semiring $M$. Then $(f, A) \cap(g, B)$ is a fuzzy soft bi-ideal over $M$.

Proof. By Definition 3.9, we have $(f, A) \cap(g, B)=(h, C)$ where $C=A \cup B$.

Case $(i): h_{c}=f_{c}$ if $c \in A \backslash B$. Then $h_{c}$ is a fuzzy bi- ideal of $M$ since $(f, A)$ is a fuzzy soft bi-ideal over $M$.

Case (ii) : If $c \in B \backslash A$ then $h_{c}=g_{c}$. Therefore $h_{c}$ is a fuzzy bi-ideal of $M$ since $(g, B)$ is a fuzzy soft ideal over $M$.

Case (iii) : If $c \in A \cap B$, and $x, y z \in S, \alpha \in \Gamma$ then $h_{c}=f_{c} \cap g_{c}$ and

$$
\begin{array}{rlr}
h_{c}(x+y) & = & \min \left\{f_{c}(x+y), g_{c}(x+y)\right\} \\
& \geq & \min \left\{\min \left\{f_{c}(x), f_{c}(y)\right\}, \min \left\{g_{c}(x), g_{c}(y)\right\}\right\} \\
& =\min \left\{\min \left\{f_{c}(x), g_{c}(x)\right\}, \min \left\{f_{c}(y), g_{c} z(y)\right\}\right\} \\
& = & \min \left\{f_{c} \cap g_{c}(x), f_{c} \cap g_{c}(y)\right\} \\
& = & \min \left\{h_{c}(x), h_{c}(y)\right\}
\end{array}
$$

and

$$
\begin{array}{rlc}
h_{c}(x \alpha z \beta y) & = & \min \left\{f_{c}(x \alpha z \beta y), g_{c}(x \alpha z \beta y)\right\} \\
& \geq \min \left\{\max \left\{f_{c}(x), f_{c}(y)\right\}, \max \left\{g_{c}(x), g_{c}(y)\right\}\right\} \\
& =\max \left\{\min \left\{f_{c}(x), g_{c}(x)\right\}, \min \left\{f_{c}(y), g_{c}(y)\right\}\right\} \\
& = & \max \left\{f_{c} \cap g_{c}(x), f_{c} \cap g_{c}(y)\right\} \\
& = & \max \left\{h_{c}(x), h_{c}(y)\right\}
\end{array}
$$

Let $x, y \in S$ and $x \leq y$. Then

$$
\begin{aligned}
& f_{c}(x) \geq f_{c}(y), g_{c}(x) \geq g_{c}(y) \\
\Rightarrow & \min \left(f_{c}(x), g_{c}(x)\right) \geq \min \left(f_{c}(y), g_{c}(y)\right) \\
\Rightarrow & f_{c} \cap g_{c}(x) \geq f_{c} \cap g_{c}(y) . \\
\Rightarrow & h_{c}(x) \geq h_{c}(y) .
\end{aligned}
$$

Hence $h_{c}$ is a fuzzy bi- ideal of $M$. Thus $(f, A) \cap(g, B)$ is a fuzzy soft bi- ideal over $M$.

Theorem 3.25. Let $(f, A)$ and $(g, B)$ be fuzzy soft interior-ideals over ordered $\Gamma$-semiring M. Then $(f, A) \cap(g, B)$ is a fuzzy soft interior-ideal over $M$.

Proof. By Definition 3.9, we have $(f, A) \cap(g, B)=(h, C)$ where $C=A \cup B$.

Case $(i): h_{c}=f_{c}$ if $c \in A \backslash B$. Then $h_{c}$ is a fuzzy interior- ideal of $M$ since $(f, A)$ is a fuzzy 
soft bi-ideal over $M$.

Case (ii) : If $c \in B \backslash A$ then $h_{c}=g_{c}$. Therefore $h_{c}$ is a fuzzy interior- ideal of $M$ since $(g, B)$ is a fuzzy soft interior- ideal over $M$.

Case (iii) : If $c \in A \cap B$, and $x, y, z \in M, \alpha \in \Gamma$ then $h_{c}=f_{c} \cap g_{c}$ and

$$
\begin{array}{rlc}
h_{c}(x+y) & = & \min \left\{f_{c}(x+y), g_{c}(x+y)\right\} \\
& \geq \min \left\{\min \left\{f_{c}(x), f_{c}(y)\right\}, \min \left\{g_{c}(x), g_{c}(y)\right\}\right\} \\
& =\min \left\{\min \left\{f_{c}(x), g_{c}(x)\right\}, \min \left\{f_{c}(y), g_{c}(y)\right\}\right\} \\
& = & \min \left\{f_{c} \cap g_{c}(x), f_{c} \cap g_{c}(y)\right\} \\
& = & \min \left\{h_{c}(x), h_{c}(y)\right\}
\end{array}
$$

and

$$
\begin{array}{rlrl}
h_{c}(x \alpha z \beta y) & = & \min \left\{f_{c}(x \alpha z \beta y), g_{c}(x \alpha z \beta y)\right\} \\
& \geq & \left.\left.\min \left\{f_{c}(z)\right)\right\},\left\{g_{c}(z)\right\}\right\} \\
& = & & \left\{\min \left\{f_{c}(z), g_{c}(z)\right\}\right\} \\
& = & f_{c} \cap g_{c}(z) \\
& = & h_{c}(z)
\end{array}
$$

Let $x, y \in M$ and $x \leq y$. Then

$$
\begin{aligned}
& f_{c}(x) \geq f_{c}(y), g_{c}(x) \geq g_{c}(y) \\
\Rightarrow & \min \left(f_{c}(x), g_{c}(x)\right) \geq \min \left(f_{c}(y), g_{c}(y)\right) \\
\Rightarrow & f_{c} \cap g_{c}(x) \geq f_{c} \cap g_{c}(y) . \\
\Rightarrow & h_{c}(x) \geq h_{c}(y) .
\end{aligned}
$$

Hence $h_{c}$ is a fuzzy interior- ideal of $M$. Thus $(f, A) \cap(g, B)$ is a fuzzy soft interior- ideal over $M$.

Theorem 3.26. Let $(f, A)$ and $(g, B)$ be fuzzy soft ideals over ordered $\Gamma$-semiringM. Then $(f, A) \cup(g, B)$ is a fuzzy soft ideal over $M$.

Proof. By Definition 3.10, we have $(f, A) \cup(g, B)=(h, C)$ where $C=A \cup B$

$$
h_{c}= \begin{cases}f_{c}, & \text { if } c \in A \backslash B ; \\ g_{c}, & \text { if } c \in B \backslash A ; \\ f_{c} \cup g_{c}, & \text { if } c \in A \cap B .\end{cases}
$$

Case(i): If $c \in A \backslash B$ then $h_{c}=f_{c}, h_{c}$ is a fuzzy ideal of $M$ since $(f, A)$ is a fuzzy soft ideal over $M$.

Case(ii): If $c \in B \backslash A$ then $h_{c}=g_{c}, h_{c}$ is a fuzzy ideal of $M$ since $(g, B)$ is a fuzzy soft ideal over $M$.

Case(iii): If $c \in A \cap B$ then for all $x, y \in M, \alpha \in \Gamma$,

$h_{c}(x)=f_{c} \cup g_{c}(x)=\max \left\{f_{c}(x), g_{c}(x)\right\}$ 


$$
\begin{array}{rlc}
h_{c}(x+y) & = & \max \left\{f_{c}(x+y), g_{c}(x+y)\right\} \\
& \geq & \max \left\{\min \left\{f_{c}(x), f_{c}(y)\right\}, \min \left\{g_{c}(x), g_{c}(y)\right\}\right\} \\
& = & \min \left\{\max \left\{f_{c}(x), g_{c}(x)\right\}, \max \left\{f_{c}(y), g_{c}(y)\right\}\right\} \\
& = & \min \left\{(f \cup g)_{c}(x),(f \cup g)_{c}(y)\right\} \\
h_{c}(x \alpha y) & = & (f \cup g)_{c}(x \alpha y) \\
& = & \max \left\{f_{c}(x \alpha y), g_{c}(x \alpha y)\right\} \\
\geq & \max \left\{\max \left\{f_{c}(x), f_{c}(y)\right\}, \max \left\{g_{c}(x), g_{c}(y)\right\}\right\} \\
& =\max \left\{\max \left\{f_{c}(x), g_{c}(x)\right\}, \max \left\{f_{c}(y), g_{c}(y)\right\}\right\}
\end{array}
$$

Let $x, y \in S$ and $x \leq y$. Then

$$
\begin{aligned}
& f_{c}(x) \geq f_{c}(y), g_{c}(x) \geq g_{c}(y) \\
\Rightarrow & \max \left(f_{c}(x), g_{c}(x)\right) \geq \max \left(f_{c}(y), g_{c}(y)\right) \\
\Rightarrow & f_{c} \cup g_{c}(x) \geq f_{c} \cup g_{c}(y) . \\
\Rightarrow & h_{c}(x) \geq h_{c}(y) .
\end{aligned}
$$

Hence $h_{c}$ is a fuzzy ideal of $M$. Therefore $(h, C)$ is a fuzzy soft ideal over $M$.

Theorem 3.27. Let $(f, A)$ and $(g, B)$ be fuzzy soft bi-ideals over ordered $\Gamma$-semiringM. Then $(f, A) \cup(g, B)$ is a fuzzy soft bi-ideal over $M$.

Proof. By Definition 3.10, we have $(f, A) \cup(g, B)=(h, C)$ where $C=A \cup B$

$$
h_{c}= \begin{cases}f_{c}, & \text { if } c \in A \backslash B \\ g_{c}, & \text { if } c \in B \backslash A ; \\ f_{c} \cup g_{c}, & \text { if } c \in A \cap B\end{cases}
$$

Case(i): If $c \in A \backslash B$ then $h_{c}=f_{c}, h_{c}$ is a fuzzy ideal of $M$ since $(f, A)$ is a fuzzy soft ideal over $M$.

Case(ii): If $c \in B \backslash A$ then $h_{c}=g_{c}, h_{c}$ is a fuzzy ideal of $M$ since $(g, B)$ is a fuzzy soft ideal over $M$.

Case(iii): If $c \in A \cap B$ then for all $x, y, z \in S, \alpha \in \Gamma$,

$$
\begin{aligned}
& h_{c}(x)=f_{c} \cup g_{c}(x)=\max \left\{f_{c}(x), g_{c}(x)\right\} \\
& h_{c}(x+y)=\quad \max \left\{f_{c}(x+y), g_{c}(x+y)\right\} \\
& \geq \max \left\{\min \left\{f_{c}(x), f_{c}(y)\right\}, \min \left\{g_{c}(x), g_{c}(y)\right\}\right\} \\
& =\min \left\{\max \left\{f_{c}(x), g_{c}(x)\right\}, \max \left\{f_{c}(y), g_{c}(y)\right\}\right\} \\
& =\min \left\{(f \cup g)_{c}(x),(f \cup g)_{c}(y)\right\}
\end{aligned}
$$




$$
\begin{array}{rlrl}
h_{c}(x \alpha z \beta y) & = & \max \left\{f_{c}(x \alpha z \beta y), g_{c}(x \alpha z \beta y)\right\} \\
& \geq \max \left\{\max \left\{f_{c}(x), f_{c}(y)\right\}, \max \left\{g_{c}(x), g_{c}(y)\right\}\right\} \\
& =\max \left\{\min \left\{f_{c}(x), g_{c}(x)\right\}, \min \left\{f_{c}(y), g_{c}(y)\right\}\right\} \\
& = & \max \left\{f_{c} \cap g_{c}(x), f_{c} \cap g_{c}(y)\right\} \\
& = & \max \left\{h_{c}(x), h_{c}(y)\right\}
\end{array}
$$

Hence $h_{c}$ is a fuzzy bi- ideal of $M$. Therefore $(h, C)$ is a fuzzy soft bi- ideal over $M$.

Theorem 3.28. Let $(f, A)$ and $(g, B)$ be two fuzzy soft interor ideals over ordered $\Gamma$-semiringM. Then $(f, A) \cup(g, B)$ is a fuzzy soft interior ideal over $M$.

Proof. By Definition 3.10, we have $(f, A) \cup(g, B)=(h, C)$ where $C=A \cup B$

$$
h_{c}= \begin{cases}f_{c}, & \text { if } c \in A \backslash B ; \\ g_{c}, & \text { if } c \in B \backslash A ; \\ f_{c} \cup g_{c}, & \text { if } c \in A \cap B .\end{cases}
$$

Case(i): If $c \in A \backslash B$ then $h_{c}=f_{c}, h_{c}$ is a fuzzy ideal of $M$ since $(f, A)$ is a fuzzy soft ideal over $M$.

Case(ii): If $c \in B \backslash A$ then $h_{c}=g_{c}, h_{c}$ is a fuzzy ideal of $M$ since $(g, B)$ is a fuzzy soft ideal over $M$.

Case(iii): If $c \in A \cap B$ then for all $x, y, z \in M, \alpha \in \Gamma$,

$$
\begin{aligned}
& h_{c}(x)=f_{c} \cup g_{c}(x)=\max \left\{f_{c}(x), g_{c}(x)\right\} \\
& h_{c}(x+y)=\quad \max \left\{f_{c}(x+y), g_{c}(x+y)\right\} \\
& \geq \max \left\{\min \left\{f_{c}(x), f_{c}(y)\right\}, \min \left\{g_{c}(x), g_{c}(y)\right\}\right\} \\
& =\min \left\{\max \left\{f_{c}(x), g_{c}(x)\right\}, \max \left\{f_{c}(y), g_{c}(y)\right\}\right\} \\
& =\min \left\{(f \cup g)_{c}(x),(f \cup g)_{c}(y)\right\} \\
& h_{c}(x \alpha z \beta y)=\min \left\{f_{c}(x \alpha z \beta y), g_{c}(x \alpha z \beta y)\right\} \\
& \left.\left.\geq \min \left\{f_{c}(z)\right)\right\},\left\{g_{c}(z)\right\}\right\} \\
& =\quad\left\{\min \left\{f_{c}(z), g_{c}(z)\right\}\right\} \\
& =\quad\left\{f_{c} \cap g_{c}(z)\right\} \\
& =\quad\left\{h_{c}(x), h_{c}(y)\right\}
\end{aligned}
$$

Let $x, y \in S$ and $x \leq y$. Then

$$
\begin{aligned}
& f_{c}(x) \geq f_{c}(y), g_{c}(x) \geq g_{c}(y) \\
\Rightarrow & \max \left(f_{c}(x), g_{c}(x)\right) \geq \max \left(f_{c}(y), g_{c}(y)\right) \\
\Rightarrow & f_{c} \cup g_{c}(x) \geq f_{c} \cup g_{c}(y) . \\
\Rightarrow & h_{c}(x) \geq h_{c}(y) .
\end{aligned}
$$

Hence $h_{c}$ is a fuzzy interior- ideal of $M$. Therefore $(h, C)$ is a fuzzy soft interior ideal over M. 
Theorem 3.29. Let $(f, A)$ and $(g, B)$ be fuzzy soft ideals over an ordered $\Gamma$-semiring $M$. Then $(f, A) \wedge(g, B)$ is a fuzzy soft ideal over $M$.

Proof. By Definition 3.11, $(f, A) \wedge(g, B)=(h, C)$ where $C=A \times B$.

Let $c=(a, b) \in C=A \times B$ and $x, y \in M, \alpha \in \Gamma$. Then

$$
\begin{aligned}
h_{c}(x+y) & =f_{a}(x+y) \wedge g_{b}(x+y) \\
& =\min \left\{f_{a}(x+y), g_{b}(x+y)\right\} \\
& \geq \min \left\{\min \left\{f_{a}(x), f_{a}(y)\right\}, \min \left\{g_{b}(x), g_{b}(y)\right\}\right\} \\
& =\min \left\{\min \left\{f_{a}(x), g_{b}(x)\right\}, \min \left\{f_{a}(y), g_{b}(y)\right\}\right\} \\
& =\min \left\{f_{a} \wedge g_{b}(x), f_{a} \wedge g_{b}(y)\right\} \\
& =\min \left\{h_{c}(x), h_{c}(y)\right\} \\
h_{c}(x \alpha y) & =f_{a}(x \alpha y) \wedge g_{b}(x \alpha y) \\
& =\min \left\{f_{a}(x \alpha y), g_{b}(x \alpha y)\right\} \\
& \geq \min \left\{\max \left\{f_{a}(x), f_{a}(y)\right\}, \max \left\{g_{b}(x), g_{b}(y)\right\}\right\} \\
& =\max \left\{\min \left\{f_{a}(x), g_{b}(x)\right\}, \min \left\{f_{a}(y), g_{b}(y)\right\}\right\} \\
& =\max \left\{h_{c}(x), h_{c}(y)\right\}
\end{aligned}
$$

Let $x, y \in M$ and $x \leq y$. Then

$$
\begin{aligned}
& f_{a}(x) \geq f_{a}(y), g_{b}(x) \geq g_{b}(y) \\
\Rightarrow & \min \left(f_{a}(x), g_{b}(x)\right) \geq \min \left(f_{a}(y), g_{b}(y)\right) \\
\Rightarrow & f_{a} \wedge g_{b}(x) \geq f_{a} \wedge g_{b}(y) \\
\Rightarrow & h_{c}(x) \geq h_{c}(y)
\end{aligned}
$$

Hence $h_{c}$ is a fuzzy ideal of $M$. Therefore $(h, A \times B)$ is a fuzzy soft ideal over $M$.

Theorem 3.30. Let $(f, A)$ and $(g, B)$ be fuzzy soft bi-ideals over an ordered $\Gamma$-semiring M. Then $(f, A) \wedge(g, B)$ is a fuzzy soft bi-ideal over $M$.

Proof. By Definition 3.11, $(f, A) \wedge(g, B)=(h, C)$ where $C=A \times B$.

Let $c=(a, b) \in C=A \times B$ and $x, y, z \in S, \alpha \in \Gamma$. Then

$$
\begin{aligned}
h_{c}(x+y) & =f_{a}(x+y) \wedge g_{b}(x+y) \\
& =\min \left\{f_{a}(x+y), g_{b}(x+y)\right\} \\
& \geq \min \left\{\min \left\{f_{a}(x), f_{a}(y)\right\}, \min \left\{g_{b}(x), g_{b}(y)\right\}\right\} \\
& =\min \left\{\min \left\{f_{a}(x), g_{b}(x)\right\}, \min \left\{f_{a}(y), g_{b}(y)\right\}\right\} \\
& =\min \left\{f_{a} \wedge g_{b}(x), f_{a} \wedge g_{b}(y)\right\} \\
& =\min \left\{h_{c}(x), h_{c}(y)\right\}
\end{aligned}
$$




$$
\begin{array}{rlc}
h_{c}(x \alpha z \beta y) & = & \min \left\{f_{a}(x \alpha z \beta y), g_{b}(x \alpha z \beta y)\right\} \\
& \geq \min \left\{\max \left\{f_{a}(x), f_{a}(y)\right\}, \max \left\{g_{b}(x), g_{b}(y)\right\}\right\} \\
& =\max \left\{\min \left\{f_{a}(x), g_{b}(x)\right\}, \min \left\{f_{a}(y), g_{b}(y)\right\}\right\} \\
& = & \max \left\{f_{a} \cap g_{b}(x), f_{a} c \cap g_{b}(y)\right\} \\
& = & \max \left\{h_{c}(x), h_{c}(y)\right\}
\end{array}
$$

Let $x, y \in M$ and $x \leq y$. Then

$$
\begin{aligned}
& f_{a}(x) \geq f_{a}(y), g_{b}(x) \geq g_{b}(y) \\
\Rightarrow & \min \left(f_{a}(x), g_{b}(x)\right) \geq \min \left(f_{a}(y), g_{b}(y)\right) \\
\Rightarrow & f_{a} \wedge g_{b}(x) \geq f_{a} \wedge g_{b}(y) \\
\Rightarrow & h_{c}(x) \geq h_{c}(y)
\end{aligned}
$$

Hence $h_{c}$ is a fuzzy bi- ideal of $M$. Therefore $(h, A \times B)$ is a fuzzy soft bi- ideal over $M$.

Theorem 3.31. Let $(f, A)$ and $(g, B)$ be fuzzy soft interior idealss over an ordered $\Gamma$-semiring M. Then $(f, A) \wedge(g, B)$ is a fuzzy soft interior ideal over $M$.

Proof. By Definition 3.11, $(f, A) \wedge(g, B)=(h, C)$ where $C=A \times B$.

Let $c=(a, b) \in C=A \times B$ and $x, y, z \in M, \alpha \in \Gamma$. Then

$$
\begin{aligned}
& h_{c}(x+y)=f_{a}(x+y) \wedge g_{b}(x+y) \\
&=\min \left\{f_{a}(x+y), g_{b}(x+y)\right\} \\
& \geq \min \left\{\min \left\{f_{a}(x), f_{a}(y)\right\}, \min \left\{g_{b}(x), g_{b}(y)\right\}\right\} \\
&=\min \left\{\min \left\{f_{a}(x), g_{b}(x)\right\}, \min \left\{f_{a}(y), g_{b}(y)\right\}\right\} \\
&=\min \left\{f_{a} \wedge g_{b}(x), f_{a} \wedge g_{b}(y)\right\} \\
&=\min \left\{h_{c}(x), h_{c}(y)\right\} \\
& h_{c}(x \alpha z \beta y)=\min \left\{f_{a}(x \alpha z \beta y), g_{b}(x \alpha z \beta y)\right\} \\
&\left.\geq \min \left\{f_{a}(z), g_{b}(z)\right\}\right\} \\
&= \\
& f_{a} \cap g_{b}(z) \\
& h_{c}(z)
\end{aligned}
$$

Let $x, y \in M$ and $x \leq y$. Then

$$
\begin{aligned}
& f_{a}(x) \geq f_{a}(y), g_{b}(x) \geq g_{b}(y) \\
\Rightarrow & \min \left(f_{a}(x), g_{b}(x)\right) \geq \min \left(f_{a}(y), g_{b}(y)\right) \\
\Rightarrow & f_{a} \wedge g_{b}(x) \geq f_{a} \wedge g_{b}(y) \\
\Rightarrow & h_{c}(x) \geq h_{c}(y)
\end{aligned}
$$

Hence $h_{c}$ is a fuzzy interior- ideal of $M$. Therefore $(h, A \times B)$ is a fuzzy soft ideal over $M$. 
Theorem 3.32. Let $(f, A)$ and $(g, B)$ be fuzzy soft ideals(bi-ideals,interior ideals) over an ordered $\Gamma$-semiring $M$. Then $(f, A) \vee(g, B)$ is a fuzzy soft ideal (bi-ideal,interior ideal) over $M$.

Proof. The proof is similar to that of Theorem 3.23 and using Definition 3.12.

Definition 3.33. Let $(f, A)$ be a fuzzy soft ordered $\Gamma$-semiring over $\Gamma$-semiring $M$. A non null fuzzy soft set $(g, B)$ over $M$ is called a fuzzy soft ideal of $(f, A)$ if it satisfies the following conditions

(i) $(g, B)$ is a fuzzy soft subset of $(f, A)$

(ii) $(g, B)$ is a fuzzy soft ideal over $M$.

Definition 3.34. A fuzzy set $\mu$ of an ordered $\Gamma$-semiring $M$ is said to be normal fuzzy ideal if $\mu$ is a fuzzy ideal of $M$ and $\mu(0)=1$.

Definition 3.35. Let $(f, A)$ be fuzzy soft ideal over an ordered $\Gamma$-semiringr $M$. Then $(f, A)$ is said to be normal fuzzy soft $\Gamma$-semiring if $f_{a}$ is a normal fuzzy ideal of ordered $\Gamma$-semiring over $M$, for all $a \in A$.

Theorem 3.36. If $(f, A)$ is a fuzzy soft ideal over an ordered $\Gamma$-semiring $M$ and for each $a \in A, f_{a}^{+}$is defined by $f_{a}^{+}(x)=f_{a}(x)+1-f_{a}(0)$ for all $x \in S$ then $\left(f^{+}, A\right)$ is a normal fuzzy soft ideal over an ordered $\Gamma$-semiring $M$ and $(f, A)$ is a fuzzy soft ideal of $\left(f^{+}, A\right)$.

Proof. Let $x, y \in S, \alpha \in \Gamma$ and $a \in A$. Then

$$
\begin{array}{rlc}
f_{a}^{+}(x+y) & = & f_{a}(x+y)+1-f_{a}(0) \\
& \geq & \min \left\{f_{a}(x), f_{a}(y)\right\}+1-f_{a}(0) \\
& = & \min \left\{f_{a}(x)+1-f_{a}(0), f_{a}(y)+1-f_{a}(0)\right\} \\
& = & \min \left\{f_{a}^{+}(x), f_{a}^{+}(y)\right\} \\
f_{a}^{+}(x \alpha y) & = & f_{a}(x \alpha y)+1-f_{a}(0) \\
\geq & \max \left\{f_{a}(x), f_{a}(y)\right\}+1-f_{a}(0) \\
& = & \max \left\{f_{a}(x)+1-f_{a}(0), f_{a}(y)+1-f_{a}(0)\right\} \\
& = & \max \left\{f_{a}^{+}(x), f_{a}^{+}(y)\right\}
\end{array}
$$

If $x=0$, then $f_{a}^{+}(0)=1$ and $f_{a} \subset f_{a}^{+}$.

Let $x, y \in M$ and $x \leq y$. Then

$$
\begin{aligned}
& f_{a}(x) \geq f_{a}(y), \\
\Rightarrow & \left.f_{a}(x) \geq f_{a}(y)\right) \\
\Rightarrow & \left.f_{a}(x)+1-f_{a}(0) \geq f_{a}(y)\right)+1-f_{a}(0), \\
\Rightarrow & f_{a}^{+}(x) \geq f_{a}^{+}(y) .
\end{aligned}
$$


Hence $\left(f^{+}, A\right)$ is a normal fuzzy soft ideal over an ordered $\Gamma$-semiring over $M$ and $(f, A)$ is a soft ideal of $\left(f^{+}, A\right)$.

Theorem 3.37. Let $(f, A)$ and $(g, B)$ be fuzzy soft ideals of a fuzzy soft ordered $\Gamma$-semiring $(h, C)$ over ordered $\Gamma$-semiring $M$. Then $(f, A) \cap(g, B)$ is a fuzzy soft ideal of $(h, C)$ if it is a nonnull.

Proof. By Theorem 3.21, $(f, A) \cap(g, B)$ is a fuzzy soft ideal over $M$. By Definition 3.33, $(f, A)$ and $(g, B)$ are fuzzy soft subsets of $(h, C)$. Hence $(f, A) \cap(g, B)$ is a fuzzy soft ideal of $(h, C$.)

The following theorem can be proved easily.

Theorem 3.38. Let $(f, A)$ and $(g, B)$ be fuzzy soft ideals of a fuzzy soft ordered $\Gamma$-semiring $(h, C)$ over ordered $\Gamma$-semiring $M$. Then $(f, A) \cup(g, B)$ is a fuzzy soft ideal of $(h, C)$ if it is non null.

Theorem 3.39. Let $(f, A)$ and $(g, B)$ be fuzzy soft ordered $\Gamma$-semirings over ordered $\Gamma$-semiring M. Let $\left(f_{1}, C\right)$ and $\left(g_{1}, D\right)$ be fuzzy soft ideals of $(f, A)$ and $(g, B)$ respectively. Then $\left(f_{1}, C\right) \cap\left(g_{1}, D\right)$ is a fuzzy soft ideal of $(f, A) \cap(g, B)$ if it is non null.

Proof. Since $\left(f_{1}, C\right)$ and $\left(g_{1}, D\right)$ are fuzzy soft ideals of $(f, A)$ and $(g, B)$ respectively, we have $\left(f_{1}, C\right)$ and $\left(g_{1}, D\right)$ are fuzzy soft ideals over $M$. By Theorem $3.21,\left(f_{1}, C\right) \cap\left(g_{1}, D\right)$ is a fuzzy soft ideal over $S$ and by theorem 3.9, $(f, A) \cap(g, B)$ is a fuzzy soft $\Gamma$-semiring over $M$. Hence $\left(f_{1}, C\right) \cap\left(g_{1}, D\right)$ is a fuzzy soft ideal of $(f, A) \cap(g, B)$.

Theorem 3.40. Let $M$ be a regular ordered $\Gamma$-semiring. If $\mu$ is a fuzzy bi-ideal of $M$ then $\mu$ is a fuzzy interior ideal of $M$.

Proof. Let $\mu$ be a fuzzy bi-ideal of regular ordered $\Gamma$-semiring $M, a, x \in M$ and $\alpha \in \Gamma$. Since $M$ is a regular and $a \alpha x \in M$, there exist $\gamma, \delta \in M$ and $y \in M$ such that $a \alpha x \leq a \alpha x \gamma y \delta a \alpha x$

$$
\begin{aligned}
\Rightarrow \mu(a \alpha x) & \geq \mu(a \alpha x \gamma y \delta a \alpha x) \\
& \geq \max (\mu(a), \mu(x)) .
\end{aligned}
$$

Therefore $\mu$ is a fuzzy ideal of a regular ordered $\Gamma$-semiring $M$. Then by Theorem 3.67, $\mu$ is a fuzzy interior ideal of $M$. Hence every fuzzy bi-ideal of regular ordered $\Gamma-$ semiring $M$ is a fuzzy interior ideal of $M$.

Corollary 3.41. Let $M$ be a regular ordered $\Gamma$-semiring and $(f, A)$ be fuzzy soft bi-ideal over an ordered $\Gamma$-semiring $M$. Then $(f, A)$ isl fuzzy soft interior ideal over an ordered $\Gamma$-semiring $M$. 
Theorem 3.42. Every fuzzy ideal of ordered $\Gamma$-semiring $M$ is a fuzzy interior ideal. $M$

Proof. Suppose $\mu$ is a fuzzy ideal of ordered $\Gamma$-semiring $M$.

Then $\mu(a \alpha x \beta b) \geq \mu(a \alpha x) \geq \mu(x)$, for all $a, x, b \in M, \alpha \in \Gamma$. Hence $\mu$ is a fuzzy interior ideal.

Corollary 3.43. Let $M$ be an ordered $\Gamma$-semiring and $(f, A)$ be fuzzy soft ideal over an ordered $\Gamma$-semiring $M$. Then $(f, A)$ is a fuzzy soft interior over an ordered $\Gamma$-semiring $M$.

Theorem 3.44. Let $M$ be a regular ordered $\Gamma$-semiring. If $\mu$ is a fuzzy interior ideal of $M$ then $\mu$ is a fuzzy ideal of $M$.

Proof. Suppose $\mu$ is a fuzzy interior ideal of a regular ordered $\Gamma$-semiring $M, a, b \in M$, and $\alpha \in \Gamma$..Since $M$ is a regular and $a \alpha b \in M$, there exist $\beta, \delta \in \Gamma$ and $x \in M$ such that $a \alpha b \leq a \alpha b \beta x \delta a \alpha b$

$$
\begin{aligned}
& \Rightarrow \mu(a \alpha b) \geq \mu(a \alpha b \beta(x \delta a \alpha b)) \geq \mu(b), \text { since } \mu \text { is a fuzzy interior ideal } \\
& \Rightarrow \mu(a \alpha b) \geq \min \{\mu(a), \mu(b)\} .
\end{aligned}
$$

Therefore $\mu(a \alpha b) \geq \min (\mu(a), \mu(b))$.

Hence $\mu$ is a fuzzy ideal of regular ordered $\Gamma$-semiring.

Corollary 3.45. Let $M$ be a regular ordered $\Gamma$-semiring and $(f, A)$ be fuzzy soft interior ideal over an ordered $\Gamma$-semiring $M$. Then $(f, A)$ is a fuzzy soft ideal over an ordered $\Gamma$-semiring $M$.

Definition 3.46. Let $\mu$ be a fuzzy subset of $X$ and $a \in[0,1-\sup \{\mu(x) \mid x \in X\}], b \in[0,1]$. The mappings

$$
\mu_{a}^{T}: X \rightarrow[0,1], \mu_{b}^{M}: X \rightarrow[0,1], \text { and } \mu_{b, a}^{M T}: X \rightarrow[0,1]
$$

are called fuzzy translation, fuzzy multiplication and fuzzy magnified translation of $\mu$ respectively if

$$
\mu_{a}^{T}(x)=\mu(x)+a, \mu_{b}^{M}=b \mu(x), \mu_{b, a}^{M T}=b \mu(x)+a, \text { for all } x \in X .
$$

Theorem 3.47. A fuzzy subset $\mu$ is an interior ideal of an ordered $\Gamma$-semiring $M$ if and only if $\mu_{a}^{T}$, the fuzzy translation of $\mu$ is an interior ideal of an ordered $\Gamma-$ semiring $M$.

Proof. Suppose $\mu$ is an interior ideal of an ordered $\Gamma$-semiring $M$ and $x, y \in M, \alpha \in \Gamma$. Then 


$$
\begin{aligned}
\mu_{a}^{T}(x+y) & =\mu(x+y)+a \\
& \geq \min (\mu(x), \mu(y))+a \\
& =\min (\mu(x)+a, \mu(y)+a) \\
& =\min \left(\mu_{a}^{T}(x), \mu_{a}^{T}(y)\right) . \\
\mu_{a}^{T}(x \alpha y \beta z) & =\mu(x \alpha y \beta z)+a \\
& \geq \mu(y)+a \\
& =\mu_{a}^{T}(y) .
\end{aligned}
$$

Suppose $x, y \in M$ and $x \leq y$. Then $\mu(x) \geq \mu(y)$ and hence $\mu(x)+a \geq \mu(y)+a$. Therefore $\mu_{a}^{T}(x) \geq \mu_{a}^{T}(y)$. Hence $\mu_{a}^{T}$ is an interior ideal.

Converse is obvious

Theorem 3.48. A fuzzy subset $\mu$ is a bi-ideal of $M$ if and only if $\mu_{a}^{T}$, the fuzzy translation of $\mu$ is a bi-ideal of an ordered $\Gamma$-semiring $M$.

Proof. Suppose $\mu$ is a bi-ideal of ordered $\Gamma$-semiring $M$ and $x, y \in M, \alpha \in \Gamma$. Then

$$
\begin{aligned}
\mu_{a}^{T}(x+y) & =\mu(x+y)+a \\
& \geq \min (\mu(x), \mu(y))+a \\
& =\min (\mu(x)+a, \mu(y)+a) \\
& =\min \left(\mu_{a}^{T}(x), \mu_{a}^{T}(y)\right) . \\
\mu_{a}^{T}(x \alpha y \beta z) & \geq \max (\mu(x), \mu(y))+a \\
& =\min (\mu(x)+a, \mu(y)+a) \\
& =\min \left(\mu_{a}^{T}(x), \mu_{a}^{T}(y)\right) .
\end{aligned}
$$

Suppose $x, y \in M$ and $x \leq y$. Then $\mu(x) \geq \mu(y)$ and hence $\mu(x)+a \geq \mu(y)+a$. Therefore $\mu_{a}^{T}(x) \geq \mu_{a}^{T}(y)$. Hence $\mu_{a}^{T}$ is a bi-ideal. Converse is obvious

Corollary 3.49. Let $M$ be an ordered $\Gamma$-semiring. If $f$ is a fuzzy bi-ideal (interor ideal)of ordered $\Gamma$-semiring then $(f, A)$ is fuzzy translational bi-ideal (interor ideal) over an ordered $\Gamma$-semiring $M$, where $A=\{a \mid a \in[0,1-\sup \{\mu(x) \mid x \in X\}]\}$,

Definition 3.50. Let $f$ be fuzzy multiplicational bi-ideal (interor ideal) of an ordered $\Gamma$-semiring M. Then $(f, A)$ is said to be fuzzy multiplicational soft bi-ideal multiplicational (interor ideal) over $M$. if $f_{a}$ is multiplicational fuzzy bi-ideal (interor ideal) of ordered $\Gamma$-semiring over $M$, for all $a \in A$. where $A=\{a \mid a \in[0,1]\}$,

Corollary 3.51. Let $f$ be fuzzy translational bi-ideal over an ordered $\Gamma$-semiring $M$. Then $(f, A)$ is a fuzzy translational soft bi- ideal over $M$. where $A=\{a \mid a \in[0,1]\}$.

Theorem 3.52. Let $M$ be an ordered $\Gamma$-semiring. Then $\mu$ is a bi-ideal of $M$ if and only if $\mu_{b}^{M}$, the fuzzy multiplicational of $\mu$ is a bi-ideal of an ordered $\Gamma$-semiring $M$. 
Proof. Suppose $\mu$ is a bi-ideal of an ordered $\Gamma$-semiring $M x, y, z \in M, \alpha \in \Gamma$.

$$
\begin{aligned}
\mu_{b}^{M}(x+y) & =b \mu(x+y) \\
& \geq \operatorname{bmin}(\mu(x), \mu(y)) \\
& =\min (b \mu(x), b \mu(y)) \\
& =\min \left(\mu_{b}^{M}(x), \mu_{b}^{M}(y)\right) . \\
\mu_{b}^{M}(x \alpha y \beta z) & =b \mu(x \alpha y \beta z) \\
& \geq \operatorname{bmax}(\mu(x), \mu(z)) \\
& =\max (b \mu(x), b \mu(z)) \\
& =\max \left(\mu_{b}^{M}(x), \mu_{b}^{M}(z)\right) .
\end{aligned}
$$

If $x \leq y$ then $\mu(x) \geq \mu(y)$ and hence $b \mu(x) \geq b \mu(y)$. Therefore $\mu_{b}^{M}(x) \geq \mu_{b}^{M}(y)$. Hence $\mu_{b}^{M}$ is a bi-ideal of ordered $\Gamma$-semiring $M$. Converse is obvious.

Corollary 3.53. Let $f$ be fuzzy bi-ideal over an ordered $\Gamma$-semiringr $M$. Then $(f, A)$ is a multiplicational fuzzy soft bi-ideal over $M$.

Theorem 3.54. Let $M$ be an ordered $\Gamma$-semiring. Then $\mu$ is a interior ideal of $M$ if and only if $\mu_{b}^{M}$, the fuzzy multiplication of $\mu$ is an interior ideal of an ordered $\Gamma$-semiring $M$.

Proof. Suppose $\mu$ is an interior ideal of an ordered $\Gamma$-semiring $M x, y \in M, \alpha \in \Gamma$.

$$
\begin{aligned}
\mu_{b}^{M}(x+y) & =b \mu(x+y) \\
& \geq \operatorname{bmin}(\mu(x), \mu(y)) \\
& =\min (b \mu(x), b \mu(y)) \\
& =\min \left(\mu_{b}^{M}(x), \mu_{b}^{M}(y)\right) . \\
\mu_{b}^{M}(x \alpha y \beta z) & =b \mu(x \alpha y \beta z) \\
& \geq b \mu(z) \\
& =\mu_{b}^{M}(z) .
\end{aligned}
$$

If $x \leq y$ then $\mu(x) \geq \mu(y)$ and hence $b \mu(x) \geq b \mu(y)$. Therefore $\mu_{b}^{M}(x) \geq \mu_{b}^{M}(y)$. Hence $\mu_{b}^{M}$ is an interior ideal of ordered $\Gamma$-semiring $M$. Converse is obvious.

Corollary 3.55. Let $f$ be fuzzy multiplicational interior ideal of an ordered $\Gamma-$ semiringr $M$. Then $(f, A)$ is a fuzzy multiplicational soft interior ideal over $M$. where $A=\{a \mid a \in[0,1]\}$,

Theorem 3.56. If $f$ is fuzzy interior ideal of ordered $\Gamma$-semiring $M$ then $\left(f_{a}, A\right)$ is a soft interior ideal over $M$. where $A=\{a \mid a \in[0,1]\}$ and $f_{a}$ is a non empty level subset of $f$. 
Proof. , Let $M$ be an ordered $\Gamma$-semiring.Suppose that $f_{a} \neq \emptyset, a \in[0,1]$ and $a, b \in f_{a}, \alpha \in \Gamma$. Then

$$
\begin{aligned}
& \mu(a) \geq t, \mu(b) \geq t \\
\Rightarrow & \mu(a+b) \geq \min (\mu(a), \mu(b)) \geq \min (t, t)=t \\
\Rightarrow & a+b \in \mu_{t} . \\
\text { Also, } & \mu(a \alpha b) \geq \min (\mu(a), \mu(b)) \geq \min (t, t)=t \\
\Rightarrow & a \alpha b \in \mu_{t} .
\end{aligned}
$$

Let $x, y \in M, a \in \mu_{t}, \alpha, \beta \in \Gamma$. Then $\mu(x \alpha a \beta y) \geq \mu(a) \geq t$ and hence $x \alpha a \beta y \in \mu_{t}$. Therefore $\mu_{t}$ is an interior ideal of an ordered $\Gamma$-semiring $M$.

Theorem 3.57. If $\mu$ is a fuzzy interior ideal of a simple ordered $\Gamma$-semiring $M$ then $\mu$ is a constant function.

Proof. Let $\mu$ be a fuzzy interior ideal of a simple ordered $\Gamma$-semiring $M$ and $x \in M$. Let $(M \Gamma x \Gamma M]=\{t \in M \mid t \leq y, y \in M \Gamma x \Gamma M\}$.

Then $(M \Gamma x \Gamma M]$ is an ideal of $M$. Therefore $M=(M \Gamma x \Gamma M]$.

$$
\begin{gathered}
\text { Suppose } a \in M \Rightarrow a \in(M \Gamma x \Gamma M] \\
\Rightarrow a \leq c \alpha x \beta d, c, d \in M, \alpha, \beta \in \Gamma . \\
\Rightarrow \mu(a) \geq \mu(\operatorname{cox} \beta d) \geq \mu(x) .
\end{gathered}
$$

Similarly we can prove $\mu(x) \geq \mu(a)$. Therefore $\mu(x)=\mu(a)$. Hence the Theorem.

Corollary 3.58. Let $f$ be fuzzy multiplicational interior ideal of an ordered simple $\Gamma$-semiringr S. Then $(f, A)$ is a fuzzy soft constant function over $S$. where $A=\{a \mid a \in[0,1]\}$,

Theorem 3.59. et $M$ be an ordered $\Gamma$-semiring. Then $\mu$ is a fuzzy bi-ideal (interior ideal) of an ordered $\Gamma$-semiring $M$ if and only if $\mu_{b, a}^{M T}$, fuzzy magnified translation of $\mu$ is a fuzzy bi-ideal (interior ideal)l of $M$,

Proof. Suppose $\mu$ is a fuzzy bi-ideal (interior ideal) of $M$ $\Leftrightarrow \mu_{b}^{M}$ is a fuzzy bi-ideal (interior ideal)l of ordered $\Gamma$-semiring $M$, by Theorem ?? . $\Leftrightarrow \mu_{b, a}^{M T}$ is a fuzzy bi-ideal (interior ideal) of $M$, by Theorem 3.48 .

Hence the Theorem.

Corollary 3.60. Let $f$ be fuzzy magnified translational bi-ideal (interior ideal) of an ordered $\Gamma$-semiringr $M$. Then $(f, A)$ is a fuzzy magnified translational bi-ideal (interior ideal) over M.where $A=\{a \mid a \in[0,1-\sup \{\mu(x) \mid x \in S\}]\}$, 
Theorem 3.61. Let $M$ be an ordered $\Gamma$-semiring, $E$ be a parameterset and $A, B \subseteq E$. If $(f, A)$ is a fuzzy soft right ideal and $(g, B)$ is a fuzzy soft left ideal over $M$ then $(f, A) \cap(g, B)$ is a fuzzy soft quasi ideal over $M$.

Proof. By Definition 3.9, we have $(f, A) \cap(g, B)=(h, C)$ where $C=A \cup B$.

Case $(i): h_{c}=f_{c}$ if $c \in A \backslash B$. Then $h_{c}$ is a fuzzy quasi ideal of $S$ since $(f, A)$ is a fuzzy soft quasi ideal over $M$. by Theorem

Case (ii) : If $c \in B \backslash A$ then $h_{c}=g_{c}$. Therefore $h_{c}$ is a fuzzy quasi ideal of $M$ since $(g, B)$ is a fuzzy soft quasi ideal over $M$. by Theorem

Case (iii) : If $c \in A \cap B$, and $x, y \in M, \alpha \in \Gamma$ then $h_{c}=f_{c} \cap g_{c}$.

Suppose $(f, A)$ is a fuzzy soft right ideal over $M$. Then, for each $a \in A, f_{a}$ is a fuzzy right ideal of $M$. and $(g, B)$ is a fuzzy soft left ideal over $M$ then for each $b \in B, g_{b}$ is a fuzzy left ideal of $M$.

$$
\begin{aligned}
& \mu \cap \lambda(x+y)=\min \{\mu(x+y), \lambda(x+y)\} \\
& \geq \min \{\min \{\mu(x), \mu(y)\}, \min \{\lambda(x), \lambda(y)\}\} \\
& \text {. }=\min \{\min \{\mu(x), \lambda(x)\}, \min \{\mu(y), \lambda(y)\}\} \\
& =\min \{\mu \cap \lambda(x), \mu \cap \lambda(y)\} \\
& \Rightarrow f_{a}(x) \geq \sup _{(y, z) \in M_{x}}\left\{\min \left(f_{a}(u), \chi_{M}(v)\right)\right\} \\
& =f_{a} \circ \chi_{M}(x) . \\
& \geq \min \left(f_{a} \circ \chi_{M}(x), \chi_{M} \circ f_{a}(x)\right) \\
& =\chi_{M} \circ f_{a} \wedge f_{a} \circ \chi_{M}(x) \text {. } \\
& \text { Therefore } g_{b}(x) \geq \min \left\{g_{b} \circ \chi_{M}(x), \chi_{M} \circ g_{b}(x)\right\} \\
& \geq \min \left(g_{b} \circ \chi_{M}(x), \chi_{M} \circ g_{b}(x)\right) \\
& =\chi_{M} \circ g_{b} \wedge g_{b} \circ \chi_{M}(x) \text {. }
\end{aligned}
$$

Let $x, y \in M$ and $x \leq y$. Then

$$
\begin{aligned}
& f_{a}(x) \geq f_{a}(y), g_{b}(x) \geq g_{b}(y) \\
\Rightarrow & T\left(f_{a}(x), g_{b}(x)\right) \geq T\left(f_{a}(y), g_{b}(y)\right) \\
\Rightarrow & f_{a} \wedge g_{b}(x) \geq f_{a} \wedge g_{b}(y) .
\end{aligned}
$$


Therefore $f_{a} \cap g_{b}$ is a fuzzy quasi ideal of ordered $\Gamma$-semiring $M$. Hence $(f, A) \cap(g, B)$ is a fuzzy soft quasi ideal over $M$.

Theorem 3.62. If $\mu$ and $\lambda$ are fuzzy quasi ideals of an ordered $\Gamma$-semiring $M$. then $\mu \cap \lambda$ is a fuzzy bi-quasi ideal of $\Gamma$ - semigroup $M$.

Proof. Let $\mu$ and $\lambda$ be fuzzy quasi ideals of an ordered $\Gamma$-semiring $M$. Then

$$
\begin{aligned}
& \mu \cap \lambda(x+y)=\min \{\mu(x+y), \lambda(x+y)\} \\
& \geq \min \{\min \{\mu(x), \mu(y)\}, \min \{\lambda(x), \lambda(y)\}\} \\
& =\min \{\min \{\mu(x), \lambda(x)\}, \min \{\mu(y), \lambda(y)\}\} \\
& =\min \{\mu \cap \lambda(x), \mu \cap \lambda(y)\} \\
& \chi_{M} \circ \mu \cap \lambda(x)=\sup _{x=a \alpha b} \min \left\{\chi_{M}(a), \mu \cap \lambda(b)\right\} \\
& =\sup _{x=a \alpha b} \min \left\{\chi_{M}(a), \min \{\mu(b), \lambda(b)\}\right\} \\
& =\sup _{x=a a b} \min \left\{\min \left\{\chi_{M}(a), \mu(b)\right\}, \min \left\{\chi_{M}(a), \lambda(b)\right\}\right\} \\
& =\min \left\{\sup _{x=a \alpha b} \min \left\{\chi_{M}(a), \mu(b)\right\}, \sup _{x=a \alpha b} \min \left\{\chi_{M}(a), \lambda(b)\right\}\right\} \\
& =\min \left\{\chi_{M} \circ \mu(x) \cdot \chi_{M} \circ \lambda(x)\right\} \\
& =\chi_{M} \circ \mu \cap \chi_{M} \circ \lambda(x)
\end{aligned}
$$

Therefore $\chi_{M} \circ \mu \cap \lambda=\chi_{M} \circ \mu \cap \chi_{M} \circ \lambda$.

Similarly $\mu \cap \lambda \circ \chi_{M}=\mu \circ \chi_{M} \cap \lambda \circ \chi_{M}$.

Hence $\chi_{M} \circ \mu \cap \lambda \cap \mu \cap \lambda \circ \chi_{M}=\left(\chi_{M} \circ \mu\right) \cap\left(\mu \circ \chi_{M}\right) \cap\left(\chi_{M} \circ \lambda\right) \cap\left(\lambda \circ \chi_{M}\right) \subseteq \mu \cap \lambda$. Hence $\mu \cap \lambda$ is a fuzzy quasi ideal of $M$. Hence the theorem.

Corollary 3.63. Let $M$ be an ordered $\Gamma$-semiring and $E$ be a parameterset and $A, B \subseteq E$. If $(f, A)$ and $(g, B)$ are fuzzy soft quasi ideals over $M$ then $(f, A) \cap(g, B)$ is a fuzzy soft quasi ideal over $M$.

Theorem 3.64. Every fuzzy ideal of an ordered $\Gamma$-semiring $M$ is a fuzzy bi-ideal of an ordered $\Gamma$-semiring $M$.

Proof. Let $\mu$ be a fuzzy ideal of an ordered $\Gamma$-semiring $M$ and $x, y, z \in M, \alpha, \beta \in \Gamma$. Then $\mu(x \alpha y \beta z) \geq \max (\mu(x), \mu(y \beta z)) \geq \max (\mu(x), \mu(z))$. Therefore $\mu$ is a fuzzy bi-ideal of $M$.

Corollary 3.65. Let $M$ be an ordered $\Gamma$-semiring. If $(f, A)$ is a fuzzy soft ideal of ordered $\Gamma$-semiring then $(f, A)$ is fuzzy bi-ideal of ordered $\Gamma$-semiring over $M$, 
Theorem 3.66. Every fuzzy quasi ideal of an ordered $\Gamma$-semiring $M$ is a fuzzy bi-ideal of ordered $\Gamma$-semiring.

Proof. Let $\mu$ be a fuzzy quasi ideal of an ordered $\Gamma$-semirng $M, x, y, z \in M$ and $\alpha, \beta \in \Gamma$. Then

$$
\begin{aligned}
\mu(x \alpha y \beta z) & \geq \chi_{M} \circ \mu \wedge \mu \circ \chi_{M}(x \alpha y \beta z) \\
\Rightarrow \mu(x \alpha y \beta z) & \geq \max \left(\chi_{M} \circ \mu(x \alpha y \beta z), \mu \circ \chi_{M}(x \alpha y \beta z)\right) \\
& =\max \left(\sup _{(a, b) \in M_{x} \alpha y \beta z}\left\{\max \left(\chi_{M}(a), \mu(b)\right)\right\}, \sup _{(a, b) \in M_{x} \alpha y \beta z}\left\{\max \left(\mu(a), \chi_{M}(b)\right)\right)\right\} \\
& \geq \max \left(\max \left(\chi_{M}(x \alpha y), \mu(z)\right), \max \left(\mu(x), \chi_{M}(y \beta z)\right)\right) \\
& =\max (\max (1, \mu(z)), \max (\mu(x), 1)) \\
& =\max (\mu(z), \mu(x)) \\
& =\max (\mu(x), \mu(z)) .
\end{aligned}
$$

Hence $\mu$ is a fuzzy bi-ideal of $M$.

Corollary 3.67. Let $M$ be an ordered $\Gamma$-semiring. If $(f, A)$ is a fuzzy soft quasi ideal over ordered $\Gamma$-semiring then $(f, A)$ is fuzzy bi-ideal over ordered $\Gamma$-semiring $M$,

Theorem 3.68. Let $M$ be a regular ordered $\Gamma$-semiring. Then the following fuzzy soft ideals are equivalent.

(i) $(f, A)$ is a fuzzy soft bi-ideal .

(ii) $(f, A)$ is a fuzzy soft interior ideal.

(iii) $(f, A)$ is a fuzzy soft ideal .

(iv) $(f, A)$ is a fuzzy soft quasi ideal.

Proof. Let $M$ be a regular ordered $\Gamma$-semiring. By Corollary 3.41, (i) implies (ii). By Corollary 3.45, (ii) implies (iii) . By Corollary 3.23, (iii) implies (iv). By Corollary 3.67, (iv) implies (i). Hence the theorem.

\section{Conclusion}

In this paper, we introduced the notion of fuzzy soft ordered $\Gamma$-semiring, fuzzy soft ideal,fuzzy soft bi-ideal,fuzzy soft quasi-ideal, fuzzy soft interior ideal over ordered $\Gamma$-semiring and studied some of their algebraical properies and relations between them.

\section{REFERENCES}

[1] U.Acar, F. Koyuncu and B. Tanay, Soft sets and Soft rings, Comput. and Math. with Appl., 59(2010), 3458-3463. 
[2] T.K.Dutta, S.K. Sardar and S. Goswami. Operations on fuzzy ideals of $\Gamma$-semirings, Fuzzy Inf. Eng.,01 (2011) DOI: 10.1007/s12543-012-0115-z

[3] T.K. Dutta and S. Kar, On regular ternary semirings, Advances in algebra proc. of the ICM Satellite conference in algebra and related topics, World Sci. Publ., Singapore, (2003), 205-213.

[4] F.Feng, Y.B. Jun and X. Zhao, Soft semirings, Comput. Math. Appl. 56(2008), 2621-2628.

[5] J.Ghosh, B.Dinda and T.K. Samanta, Fuzzy soft rings and Fuzzy soft ideals, Int.J. P. App.Sc.Tech. 2(2)(2011), 66-74.

[6] R. A. Good and D. R. Hughes, Associated groups for a semigroup, Bull. Amer. Math. Soc., 58(1952), 624-625.

[7] M. Henriksen, Ideals in semirings with commutative addition, Amer. Math. Soc. Notices, 5 (1958), 321.

[8] K. Iseki, Quasi-ideals in semirings without zero, Proc. Japan Acad., 34 (1958), 79-84.

[9] K. Iseki, Ideal theory of semiring, Proc. Japan Acad., 32(1956), 554-559.

[10] K. Iseki, Ideal in semirings Proc. Japan Acad., 34(1958), 29-31.

[11] R. D. Jagatap, Y.S. Pawar, Quasi-ideals and minimal quasi-ideals in $\Gamma$-semirings, Novi Sad J. Math., 39(2) (2009), 79-87.

[12] S Lajos, On the bi-ideals in semigroups, Proc. Japan Acad., 45(1969), 710-712.

[13] S. Lajos and F. A. Szasz, On the bi-ideals in associative ring, Proc. Japan Acad., 46 (1970), 505-507.

[14] H. Lehmer ,A ternary analogue of abelian groups, Amer. J. Math., 59 (1932), 329-338.

[15] G. Lister, Ternary rings, Trans. Amer, Math. Soc., 154 (1971), 37-55.

[16] P.K.Maji, R. Biswas and A.R.Roy, Fuzzy soft sets, J. Fuzzy Math., 9(3)(2001), 589-602.

[17] D. Mandal, Fuzzy ideals and fuzzy interior ideals in ordered semirings, Fuzzy Info. Eng., 6 (2014), 101-114.

[18] D. Molodtsov, Soft set theory-First results, Comput. Math. Appl., 37(1999), 19-31.

[19] M. Murali Krishna Rao, Г-semirings-I, Southeast Asian Bull. Math., 19(1)(1995) 49-54.

[20] M. Murali Krishna Rao, Г-semiringsII, Southeast Asian Bull. Math., 21(3)(1997) 281-287

[21] M.Murali Krishna Rao, The Jacobson radical of $\Gamma$-semiring, South East Asian Bull. Math., 23(1999)127134

[22] M. Murali Krishna Rao, Fuzzy soft $\Gamma$-semiring and fuzzy soft $k$ ideal over $\Gamma$-semiring, Ann. Fuzzy Math. Inform. 9 (2) (2015) 12-25.

[23] Marapureddy Murali Krishna Rao,Fuzzy softГsemiring homomorphism, Ann. Fuzzy Math. Inf., 12(4) (2016), 479489.

[24] M. Murali Krishna Rao,T-fuzzy ideals in ordered semirings, Ann.Fuzzy Math. Inf. 13(2) (2017), 253276

[25] M. Murali Krishna Rao, Left bi-quasi ideals of semirings, Bull. Int. Math. Virtual Inst, 8(2018), 45-53.

[26] Marapureddy Murali Krishna Rao. Bi-quasi-ideals and fuzzy bi-quasiideals of $\Gamma$-semigroups. Bull. Int. Math. Virtual Inst., 7(2)(2017), 231-242.

[27] Marapureddy Murali Krishna Rao,On $\Gamma$-Semiring with identity, Discuss. Math. General Algebra Appl., 37 (2017) 189207.

[28] M. Murali Krishna Rao,Bi-interior ideals of semigroups, Discuss. Math. General Algebra Appl. in press.

[29] M. Murali Krishna Rao and B. Venkateswarlu, Regular $\Gamma$-incline and field $\Gamma$-semiring, Novi Sad J. of Math., 45(2)(2015), 155-171

[30] M. Murali Krishna Rao, B. Venkateswarlu and N.Rafi,Left Bi-quasi-ideals of $\Gamma$-semirings, Asia Pac. J. Math., 4(2 )(2017), 144-153. 
[31] M. Murali Krishna Rao and B. Venkateswarlu,Fuzzy soft $k$ ideals over semiring and fuzzy soft semiring homomorphism,J. Hyperstructures, 4 (2) (2015), 93-116.

[32] N. Nobusawa, On a generalization of the ring theorye, Osaka. J.Math., 1 (1964), 81 - 89.

[33] A. Rosenfeld, Fuzzy groups, J. Math. Anal. Appl. 35 (1971), 512-519.

[34] M. K. Sen, On $\Gamma$-semigroup, Proc. of Inter. Con. of Alg. and its Appl., Decker Publication, New York, (1981), 301-308.

[35] O. Steinfeld, Uher die quasi ideals, Von halbgruppend Publ. Math., Debrecen, 4 (1956), 262275.

[36] U. M. Swamy and K. L. N. Swamy, Fuzzy prime ideals of rings, J. Math. Anal. Appl., 134 (1988), 94-103.

[37] H. S. Vandiver, Note on a simple type of algebra in which cancellation law of addition does not hold, Bull. Amer. Math. Soc.(N.S.), 40 (1934), 914-920.

[38] L. A. Zadeh, Fuzzy sets, Inf. Control, 8 (1965) 338-353.

[39] L. A. Zadeh, The concept of a linguistic variable and its application to approximate reasoning, Inform. Sci. 8 (1975) 199-249. 